\title{
Validation of the German Revised Addenbrooke's Cognitive Examination for Detecting Mild Cognitive Impairment, Mild Dementia in Alzheimer's Disease and Frontotemporal Lobar Degeneration
}

\author{
P. Alexopoulos ${ }^{a, b} \quad$ A. Ebert ${ }^{c} \quad$ T. Richter-Schmidinger ${ }^{a} \quad$ E. Schöll ${ }^{b} \quad$ B. Natale ${ }^{b}$ \\ C.A. Aguilar ${ }^{\text {b, e }} \quad$ P. Gourzis ${ }^{f} \quad$ M. Weih ${ }^{a} \quad$ R. Perneczky ${ }^{b} \quad$ J. Diehl-Schmid ${ }^{b}$ \\ T. Kneib ${ }^{d}$ H. Förstl ${ }^{b} \quad$ A. Kurz ${ }^{b} \quad$ A. Danek ${ }^{c} \quad$ J. Kornhuber ${ }^{a}$ \\ a Department of Psychiatry and Psychotherapy, Friedrich-Alexander-Universität Erlangen-Nürnberg, \\ Erlangen, ${ }^{b}$ Department of Psychiatry and Psychotherapy, Technische Universität München, and \\ 'Department of Neurology, Ludwig-Maximilians-Universität München, Munich, and ${ }^{\mathrm{d}}$ Institute of Mathematics, \\ Carl-von-Ossietzky-Universität Oldenburg, Oldenburg, Germany; ${ }^{e}$ Department of Child and Adolescent Psychiatry, \\ Hospital Barros Luco Trudeau, Universidad de Santiago de Chile, Santiago de Chile, Chile; 'Department of \\ Psychiatry, University of Patras, Patras, Greece
}

\section{Key Words}

Mild cognitive impairment $\cdot$ Alzheimer's disease .

Frontotemporal lobar degeneration · Addenbrooke's

Cognitive Examination, revised edition - Mini Mental State

Examination

\begin{abstract}
Background/Aims: The diagnostic accuracy of the German version of the revised Addenbrooke's Cognitive Examination (ACE-R) in identifying mild cognitive impairment $(\mathrm{MCl})$, mild dementia in Alzheimer's disease (AD) and mild dementia in frontotemporal lobar degeneration (FTLD) in comparison with the conventional Mini Mental State Examination (MMSE) was assessed. Methods: The study encompasses 76 cognitively healthy elderly individuals, 75 patients with $\mathrm{MCl}, 56$ with $A D$ and 22 with FTLD. ACE-R and MMSE were validated against an expert diagnosis based on a comprehensive diagnostic procedure. Statistical analysis was performed using the receiver operating characteristic method and regression
\end{abstract}

analyses. Results: The optimal cut-off score for the ACE-R for detecting $\mathrm{MCl}, A D$, and FTLD was $86 / 87,82 / 83$ and $83 / 84$, respectively. ACE-R was superior to MMSE only in the detection of patients with FTLD [area under the curve (AUC): 0.97 vs. 0.92], whilst the accuracy of the two instruments did not differ in identifying $\mathrm{MCl}$ and $\mathrm{AD}$. The ratio of the scores of the memory ACE-R subtest to verbal fluency subtest contributed significantly to the discrimination between $A D$ and FTLD (optimal cut-off score: 2.30/2.31, AUC: 0.77 ), whereas the MMSE and ACE-R total scores did not. Conclusion: The German ACE-R is superior to the most commonly employed MMSE in detecting mild dementia in FTLD and in the differential diagnosis between AD and FTLD. Thus it might serve as a valuable instrument as part of a comprehensive diagnostic workup in specialist centres/clinics contributing to the diagnosis and differential diagnosis of the cause of dementia.

Copyright $\odot 2010$ S. Karger AG, Basel

\section{KARGER}

Fax +41613061234 E-Mail karger@karger.ch www.karger.com
(C) 2010 S. Karger AG, Basel

$1420-8008 / 10 / 0295-0448 \$ 26.00 / 0$

Accessible online at:

www.karger.com/dem
Dr. med. P. Alexopoulos, Klinik und Poliklinik für Psychiatrie und Psychotherapie

Klinikum rechts der Isar der Technischen Universität München

Ismaninger Strasse 22, DE-81675 Munich (Germany)

Tel. +49894140 4279, Fax +498941404923

E-Mail panos.alexopoulos@lrz.tu-muenchen.de 


\section{Introduction}

Due to the rapidly ageing population in the Western world, the incidence of age-related disorders such as dementia is increasing substantially and dramatically. Dementia is predicted to become the largest health care challenge in modern history because of its medical, social and philosophical implications [1]. An early diagnosis of dementia appears to be of great importance in order to institute appropriate medical and social interventions. Alzheimer's disease (AD) is the most common cause of dementia [2], and frontotemporal lobar degeneration (FTLD) is the second most common presenile cause of neurodegenerative dementia [3]. Depending on its cause, the clinical phenotypes are quite distinct at the stage of mild dementia. Patients with mild dementia in AD, for instance, manifest memory deficits, make repetitive statements, get lost while driving and have poor judgment, whereas subjects with mild dementia in FTLD primarily present with alterations of speech (language variant of FTLD) or with behavioural disturbances such as disinhibition and lack of social awareness (behavioural variant of FTLD) [2].

In most cases, the diagnosis of dementia is established by assessing cognitive deficits and their impact on the activities of daily living. Neuropsychological instruments enable clinicians to gather evidence of cognitive impairment, help them to detect dementia and to make a differential diagnosis. In the light of the long duration (around 1-4 h) of extensive neuropsychological testing, which limits its utility in routine dementia diagnostic workup, there is a demand for screening tests [4]. Ideally, such tests are sensitive and specific, reliable and valid, quickly administered, easily scored, and capture a broad range of cognitive abilities across various levels of difficulty, thus being efficient, not only in identifying mild dementia in $\mathrm{AD}$, but also other forms of dementia $[5,6]$.

Many screening tools for dementia which are available in German have been criticized for several shortcomings. For instance, the 7-Minute Screen is difficult to score and to interpret [7]. The DemTect [8] does not assess visuospatial ability, known to be closely associated with functional abilities [9]. The 3MS has been validated in Germany only for the identification of patients with early $\mathrm{AD}$ but not with mild cognitive impairment (MCI) or with dementias of other aetiologies [10]. The Mini Mental State Examination (MMSE) [11] is currently the most widely used measure of cognitive performance in Germany and in many other countries. It has been shown to be a useful tool for distinguishing people with dementia from cogni- tively healthy individuals and to achieve an acceptable sensitivity and specificity in detecting dementia in clinical samples [12-14]. However, several weaknesses of the MMSE have been repeatedly demonstrated, including small number of items, low level of task difficulty and likelihood of ceiling effects, narrow range of cognitive domains assessed (including overrepresentation of orientation, underrepresentation of memory tasks and absence of tasks measuring executive function), limited range of possible scores for individual items [6] and low sensitivity for the detection of patients with MCI $[15,16]$. Furthermore, the diagnostic accuracy of the MMSE depends on the patient's age, education level, and ethnicity [15].

Drawing on extensive clinical and research expertise with cognitive tests, Hodges and colleagues developed the Addenbrooke's Cognitive Examination test battery [17] and a modified version of it. Changes were introduced into the revised version of Addenbrooke's Cognitive Examination (ACE-R) [18] in order to avoid ceiling effects and to provide a more balanced contribution of the component tests to the final score. Administration of the ACE- $\mathrm{R}$ requires approximately $15 \mathrm{~min}$ and is easy to score and to interpret. It incorporates the MMSE, but elaborates on memory, language and visuospatial components, and adds component tests of verbal fluency. The memory component test assesses semantic and episodic memory. In addition to recall of 3 items taken from the MMSE, there is a name and address recall and recognition test for assessing episodic memory. Semantic memory is tested with 4 questions concerning general knowledge. The language section comprises the naming of 12 line drawings of intermediate and low familiarity, semantic comprehension, sentence comprehension, repetition of words and phrases, reading of irregular words, and writing a sentence. Executive functions are tested by letter fluency (generating words beginning with the letter $\mathrm{P}$ in $1 \mathrm{~min}$ ) and category fluency (generating names of animals in $1 \mathrm{~min}$ ). Visuospatial testing includes the copying of overlapping pentagons (from the MMSE) and of a wire cube, the drawing of a clock face, dot counting and identifying fragmented letters [18].

The maximum ACE-R score of 100 points is composed of 5 component test scores: orientation and attention (18 points), memory (26 points), verbal fluency (14 points), language (26 points) and visuospatial ability (16 points) [18]. The original validation study, which was conducted at Addenbrooke's Hospital, UK, included 241 participants. It suggested an optimal cut-off score of 88 points for identifying dementia, which was associated with high sensitivity (94\%) and specificity (89\%) [18]. 
The aim of the present study was to investigate the ability of the German version of the ACE-R to detect patients with MCI, mild dementia in $\mathrm{AD}$, and mild dementia in FTLD, and to compare its diagnostic accuracy with that of the MMSE. The ACE-R and MMSE were validated against the clinical diagnosis based on a comprehensive diagnostic workup.

\section{Methods, Study Sample and Design}

We translated and modified the instrument with advice from the authors of the original ACE-R. The modifications concerned the name and address recall and recognition tests, the semantic memory test and the repetition test. Based on the original criteria, the German addresses were chosen from common street names and from less well-known towns in order to preclude natural associations (e.g. Frauenstrasse 24, Spremberg, Brandenburg). In the semantic memory test, we replaced 'the current prime minister' and 'the woman who was prime minister' by 'the name of the current chancellor' and 'the name of the previous chancellor'. The words in the repetition test ('Hippopotamus, Exzentrizität, Unverhältnismässigkeit, Verantwortungslosigkeit' and 'oberhalb, ausserhalb und unterhalb') were selected according to the criteria used in the original English version: length, frequency and difficulty to articulate. A bilingual researcher at the University of Cambridge performed a back translation into English. Comparison of the original English version with the back translation showed that the new version was similar to the original one except for the modified items [19]. The scoring system was not changed. Like the English original, the German ACE-R can be administered in approximately $15 \mathrm{~min}$.

The validation study was carried out at 3 university memory clinics, located in Erlangen and in Munich, Germany. The examination of the participants included a history from the patient and from an informant, medical, neurological and psychiatric examination, laboratory screening, brain imaging (MRI or CT) and a neuropsychological examination based on the German version of the Consortium to Establish a Registry for Alzheimer's Disease Neuropsychological Assessment Battery (CERAD-NAB) [20], which incorporates the MMSE. The additional neuropsychological examination comprised a flexible battery (Trail Making Test [21], Bayer Activities of Daily Living Scale [22], and Neuropsychiatric Inventory [23]), the components of which varied according to the aims of the neuropsychological assessment of each patient as defined by the clinician.

The participants were German-speaking and had adequate vision and hearing, although many wore glasses and some required a hearing aid. The ACE- $\mathrm{R}$ was administered after the clinical and neuropsychological examination. The components of the ACE-R that are identical to those of the MMSE were not administered twice in order to preclude learning effects. The clinical diagnoses were established independent of the performance of participants on the ACE-R. The diagnosis of dementia and the assessment of its overall severity were based on the criteria of the ICD-10 classification system [24]. AD diagnosis was based on the criteria of the National Institute of Neurological and Communicative Disorders and Stroke-Alzheimer's Disease and Related Disorders As- sociation (NINCDS-ADRDA) for probable AD [25]. The diagnosis of FTLD was established according to the revised Lund-Manchester criteria [26]. To ensure that patients with dementia had not crossed the threshold to moderate dementia, patients with a score below 15 points on the MMSE were excluded from the study. This score has been found to discriminate mild from moderate dementia [27]. MMSE staging has been proven to be an effective clinical instrument for tracking the stages of dementia [27]. The diagnosis of MCI followed the revised consensus criteria of the International Working Group on Mild Cognitive Impairment [28]. The cognitively healthy controls were recruited among spouses and friends of patients of the 3 memory clinics. They had normal cognitive performance according to the MMSE, were independent in their activities of daily living and did not have any memory complaints. Subjects with serious medical, psychiatric or neurological disorders which could affect cognitive functioning (e.g. major depression, schizophrenia, seizure disorder, head injury) or with a score on the MMSE of $<28$ were excluded.

The study protocol was approved by the Ethics Committee of the Medical Faculty of the Friedrich-Alexander-Universität Erlangen-Nürnberg, Germany.

\section{Statistical Analyses}

Statistical analyses were implemented in PASW Statistics 17.0 for Windows (SPSS, Chicago, Ill., USA); p values of less than 0.05 were considered to indicate statistical significance.

Differences with regard to demographic variables, MMSE scores and component and total scores of the ACE-R among cognitively healthy controls and patients with MCI, AD and FTLD were tested using one-way analysis of variance. Pairwise comparisons were performed using Bonferroni's test. $\chi^{2}$ tests were employed for nominal (categorical) data. If differences attained statistical significance, a linear regression analysis was carried out to investigate possible associations between the demographic variable which varied significantly across the groups and the participants' performance in the ACE-R.

The first step of the analysis of the utility of the ACE-R and the MMSE encompassed a logistic regression analysis, assessing the extent to which ACE-R and MMSE scores predict the clinical diagnosis. ACE-R and MMSE scores were fed as explanatory variables and the clinical diagnosis as outcome variable. If the utility analysis revealed significantly higher accuracy of the ACE-R in discriminating between cognitively healthy controls and patients in comparison with the MMSE, a logistic regression analysis was carried out to assess which ACE-R component tests contributed to the higher accuracy of the instrument. The second step comprised the performance of a receiver operating characteristic (ROC) curve analysis. The area under the curve (AUC) was used as a measure of the accuracy of the ACE-R and MMSE to distinguish between the groups of participants. AUC values of less than 1.0 (perfect test) refer to excellent $(>0.9)$, good $(>0.8)$, fair $(>0.7)$ and poor $(>0.6)$ accuracy [29]. Differences between AUCs were assessed with the StAR software for the statistical comparison of ROC curves [30]. The ROC was also used to select an optimal cut-off value below which an individual has a very high chance of suffering from the aforementioned clinical syndromes and disorders. 
Table 1. Description of study sample, and component and composite mean scores \pm SD on the ACE-R and on the MMSE

\begin{tabular}{|c|c|c|c|c|}
\hline Group variable & $\begin{array}{l}\text { Cognitively } \\
\text { healthy controls }\end{array}$ & MCI & $\begin{array}{l}\text { Mild dementia } \\
\text { in } \mathrm{AD}\end{array}$ & $\begin{array}{l}\text { Mild dementia } \\
\text { in FTLD }\end{array}$ \\
\hline Number of subjects & 76 & 75 & 56 & 22 \\
\hline Age, years & $69.64 \pm 7.53$ & $67.83 \pm 8.01$ & $72.00 \pm 8.18$ & $69.64 \pm 6.18$ \\
\hline Gender:female ratio, \% & 61.5 & $40.0^{\mathrm{a}}$ & 64.2 & 40.9 \\
\hline Education, years & $11.78 \pm 2.51$ & $12.00 \pm 3.27$ & $11.02 \pm 2.63$ & $11.70 \pm 3.52$ \\
\hline MMSE score & $29.09 \pm 0.73$ & $27.29 \pm 1.82^{\mathrm{a}}$ & $23.21 \pm 3.25^{\mathrm{a}}$ & $23.45 \pm 5.54^{\mathrm{a}}$ \\
\hline ACE-R, total score & $90.37 \pm 4.99$ & $81.34 \pm 9.09^{\mathrm{a}}$ & $64.80 \pm 11.32^{\mathrm{a}}$ & $64.50 \pm 17.82^{\mathrm{a}}$ \\
\hline Orientation and attention ACE- $\mathrm{R}$ component test & $17.95 \pm 0.23$ & $17.31 \pm 0.97$ & $14.30 \pm 3.07^{\mathrm{a}}$ & $14.91 \pm 3.74^{\mathrm{a}}$ \\
\hline Memory ACE-R component test & $22.45 \pm 2.70$ & $18.04 \pm 4.75^{\mathrm{a}}$ & $10.46 \pm 5.26^{\mathrm{a}}$ & $14.14 \pm 6.24^{\mathrm{a}, \mathrm{b}}$ \\
\hline Verbal fluency ACE-R component test & $9.82 \pm 2.06$ & $7.99 \pm 2.56^{\mathrm{a}}$ & $6.21 \pm 3.60^{\mathrm{a}}$ & $4.36 \pm 2.68^{\mathrm{a}, \mathrm{b}}$ \\
\hline Language ACE-R component test & $25.13 \pm 1.25$ & $23.53 \pm 2.30^{\mathrm{a}}$ & $21.23 \pm 4.24^{\mathrm{a}}$ & $19.18 \pm 6.43^{\mathrm{a}}$ \\
\hline Visuospatial ability ACE-R component test & $15.05 \pm 1.53$ & $14.65 \pm 1.75$ & $12.59 \pm 2.98^{\mathrm{a}}$ & $11.41 \pm 3.50^{\mathrm{a}}$ \\
\hline
\end{tabular}

a Significant difference from cognitively healthy controls, $\mathrm{p}<0.05$. ${ }^{\mathrm{b}}$ Significant difference from $\mathrm{AD}$ patients, $\mathrm{p}<0.05$.

\section{Results}

The present study refers to 56 patients with mild dementia in AD, 75 patients with MCI, 22 patients with mild dementia in FTLD (14 with the frontal variant of FTLD and 8 with the language variant) and 76 cognitively healthy controls. Demographic data and MMSE and ACE-R total and component scores of the four groups of individuals are presented in table 1 . There were no statistical differences regarding age and years of education between the control group and the patient groups. The MCI group included significantly fewer female participants than the healthy controls (Fisher's exact test, $\mathrm{p}=0.015$ ). The linear regression analysis, using the ACE-R scores as dependent variable and diagnosis (MCI vs. no cognitive deficits) and gender distribution as independent factors $(\mathrm{F}=28,523, \mathrm{p}<0.0001)$, did not reveal a significant impact of gender distribution on ACE-R performance (standardized partial regression coefficient of clinical diagnosis $=-0.03, \mathrm{p}=0.72$ ), whereas the impact of the clinical diagnosis was significant (standardized partial regression coefficient of clinical diagnosis $=-0.523, \mathrm{p}<0.0001$ ). No significant differences were detected between the FTLD and the AD groups with regard to age, education and gender.

Concerning the performance on ACE-R component tests, patients with MCI had significantly lower scores on all ACE-R component tests than cognitively healthy controls except for attention/orientation and visuospatial ability (memory $\mathrm{p}<0.0001$, verbal fluency $\mathrm{p}<0.0001$, language $\mathrm{p}=0.016$ ). They also had lower total scores on
MMSE and on ACE-R in comparison with healthy controls ( $p<0.0001$ for both tests). Patients with AD and FTLD performed worse than healthy controls on all ACE- $R$ component tests, as well as on the MMSE and the ACE-R total score (all $p<0.0001$ ). Regarding differences in the performance between $\mathrm{AD}$ and FTLD patients, the former scored higher on the verbal fluency ACE-R component test $(\mathrm{p}=0.044)$ and lower on ACE-R memory tasks $(\mathrm{p}=0.008)$ compared to the latter. However, there were no differences between the two groups concerning the ACE-R total score and the rest of the cognitive domains assessed with the ACE-R.

\section{Distinction between Patients with MCI and \\ Cognitively Healthy Controls}

The logistic regression analysis (likelihood ratio $\chi^{2}=$ $73.74, \mathrm{p}<0.0001$ ) with clinical diagnosis (MCI vs. healthy controls) as dependent variable and ACE-R (regression coefficient $=-0.16, \mathrm{p}<0.0001$ ) and MMSE scores (regression coefficient $=-0.87, \mathrm{p}<0.0001$ ) as independent factors revealed that both instruments can significantly contribute to the discrimination between MCI and cognitively healthy controls. The results of the ROC analyses, which showed that the AUC of the ACE-R was slightly and not significantly $(\mathrm{p}>0.05)$ larger than that of the MMSE, are displayed in figure 1 and table 2.

\section{Distinction between Patients with Mild Dementia in $A D$ and Cognitively Healthy Controls}

The ACE-R contributed and the MMSE strongly tended to contribute significantly to the discrimination be- 


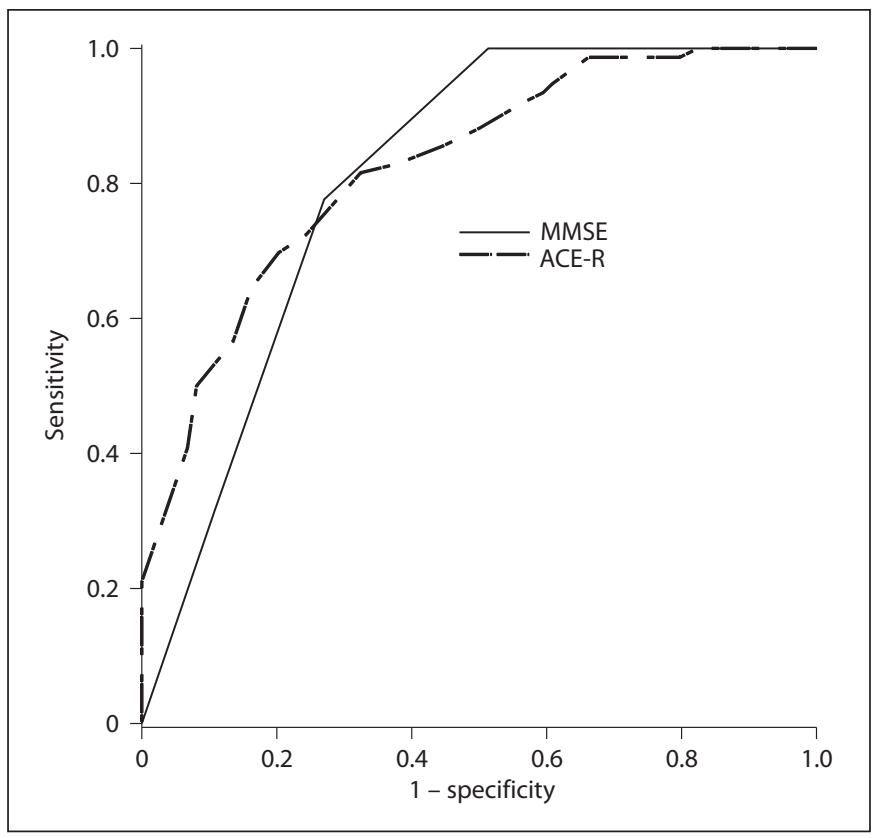

Fig. 1. ACE-R and MMSE ROC for the detection of patients with MCI.

tween $\mathrm{AD}$ and cognitively healthy controls according to the logistic regression analysis (likelihood ratio $\chi^{2}=$ $167.68, \mathrm{p}<0.0001$ ) with clinical diagnosis (AD vs. healthy controls) as dependent variable and ACE-R (regression coefficient $=-0.44, \mathrm{p}=0.02$ ) and MMSE scores (regression coefficient $=-6.48, \mathrm{p}=0.05$ ) as independent factors. The AUCs did not differ according to the ROC analyses (table 2; fig. 2).

\section{Distinction between Patients with Mild Dementia in \\ FTLD and Cognitively Healthy Controls}

The logistic regression analysis (likelihood ratio $\chi^{2}=$ 72.28, p < 0.0001) with clinical diagnosis (FTLD vs. healthy controls) as dependent variable and ACE-R (regression coefficient $=-0.28, \mathrm{p}=0.01)$ and MMSE scores (regression coefficient $=-0.85, \mathrm{p}=0.19$ ) as independent factors revealed that only the ACE-R significantly contributes to the discrimination between FTLD and cognitively healthy controls. Due to this significant difference between the accuracy of the two instruments, a further logistic regression analysis (likelihood ratio $\chi^{2}=80.8$, $\mathrm{p}<0.0001$ ) was carried out to assess which ACE-R component tests contribute significantly to the discrimination between FTLD and cognitively healthy controls. The ACE-R component tests and the clinical diagnosis (FTLD

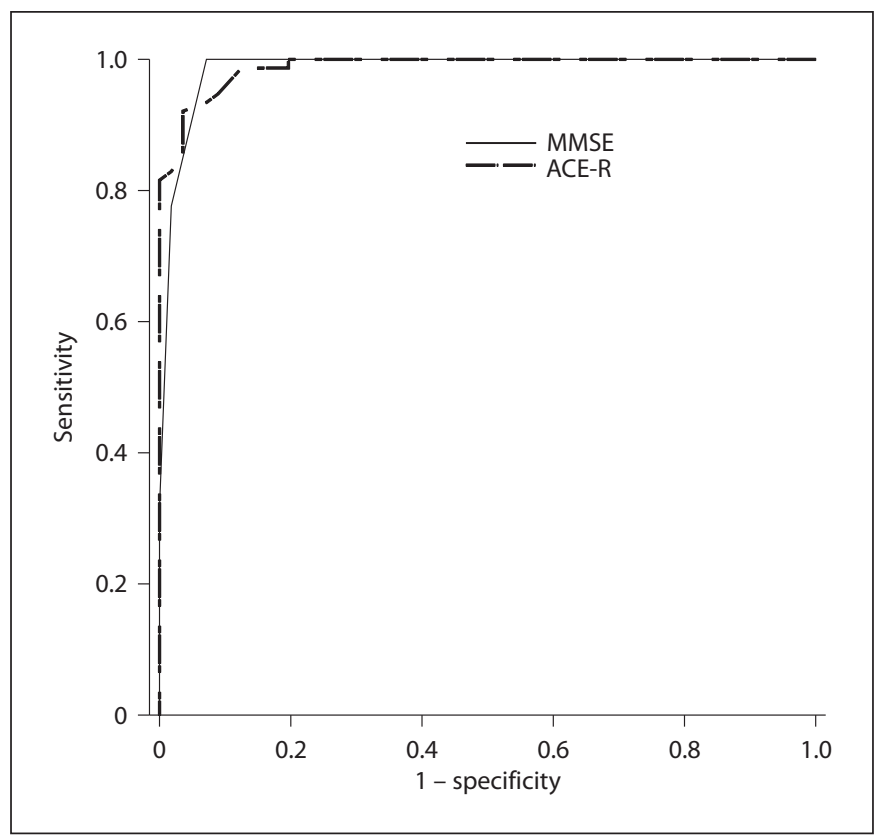

Fig. 2. ACE-R and MMSE ROC for the detection of patients with mild dementia in AD.

Table 2. Optimal cut-off scores and diagnostic utility of the ACE$\mathrm{R}$ and the MMSE for identifying MCI, mild dementia in AD and mild dementia in FTLD

\section{ACE-R MMSE}

Distinction between patients with MCI and cognitively healthy controls

$\begin{array}{lr}\text { Optimal cut-off score } \quad 86 / 87 & 28 / 29\end{array}$

$\begin{array}{lll}\text { Sensitivity } & 0.82 & 0.78\end{array}$

$\begin{array}{lll}\text { Specificity } & 0.68 & 0.73\end{array}$

Area under the curve $\quad 0.83 \quad 0.81$

Distinction between patients with mild dementia in AD and cognitively healthy controls

$\begin{array}{lll}\text { Optimal cut-off score } & 82 / 83 \quad 27 / 28\end{array}$

$\begin{array}{lll}\text { Sensitivity } & 0.92 & 1.00\end{array}$

$\begin{array}{lll}\text { Specificity } & 0.96 & 0.93\end{array}$

$\begin{array}{lll}\text { Area under the curve } & 0.99 & 0.99\end{array}$

Distinction between patients with mild dementia in FTLD and cognitively healthy controls

$\begin{array}{lrr}\text { Optimal cut-off score } & 83 / 84 & 27 / 28\end{array}$

$\begin{array}{lll}\text { Sensitivity } & 0.88 & 0.73\end{array}$

$\begin{array}{lll}\text { Specificity } & 0.96 & 0.97\end{array}$

Area under the curve $\quad 0.97 \quad 0.92$ 


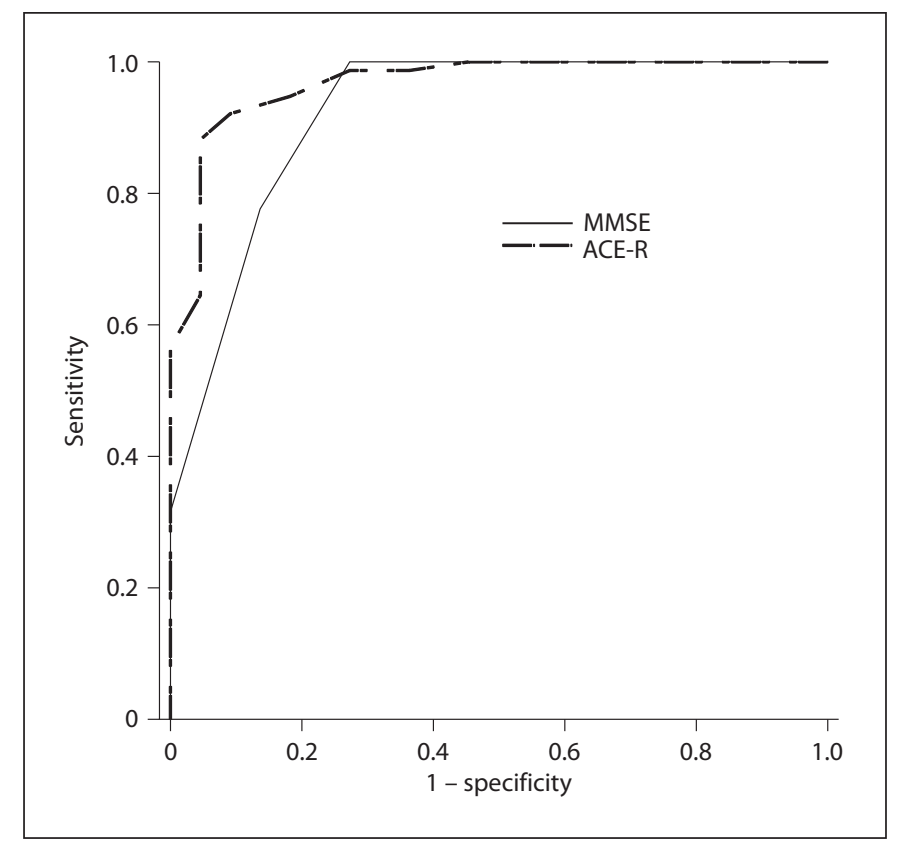

Fig. 3. ACE-R and MMSE ROC for the detection of patients with mild dementia in FTLD.

vs. healthy controls) were entered into the model as independent and dependent variables, respectively. The ACE$\mathrm{R}$ verbal fluency component test (regression coefficient $=$ $-0.73, p=0.01$ ) and the language component test (regression coefficient $=-0.92, \mathrm{p}=0.02$ ) were significantly associated with the clinical diagnosis, whereas the ACE-R memory component test (regression coefficient $=-0.24$, $\mathrm{p}=0.17$ ), the visuospatial component test (regression coefficient $=0.38, \mathrm{p}=0.37$ ) and the attention-orientation component test (regression coefficient $=-3.31, \mathrm{p}=0.07$ ) were not. The AUC of the ACE-R was larger than that of the MMSE (0.97 vs. 0.92), though the difference did not attain statistical significance $(\mathrm{p}>0.05)$ (table 2; fig. 3).

Regarding the utility of the ACE- $\mathrm{R}$ in discriminating between healthy controls and patients suffering from the frontal variant of FTLD, the results of the regression and ROC analyses did not deviate from the aforementioned findings concerning all FTLD patients. In short, the patients with the frontal variant of FTLD did not differ from healthy controls in demographic characteristics. Only the ACE- $\mathrm{R}$ (regression coefficient $=-0.27, \mathrm{p}=0.02$ ) predicted the clinical diagnosis with highly significant accuracy and not the MMSE (regression coefficient $=-0.77$, $\mathrm{p}=0.28$ ) according to the regression analysis (likelihood ratio $\left.\chi^{2}=54.27, \mathrm{p}<0.0001\right)$. The ROC also revealed a su-

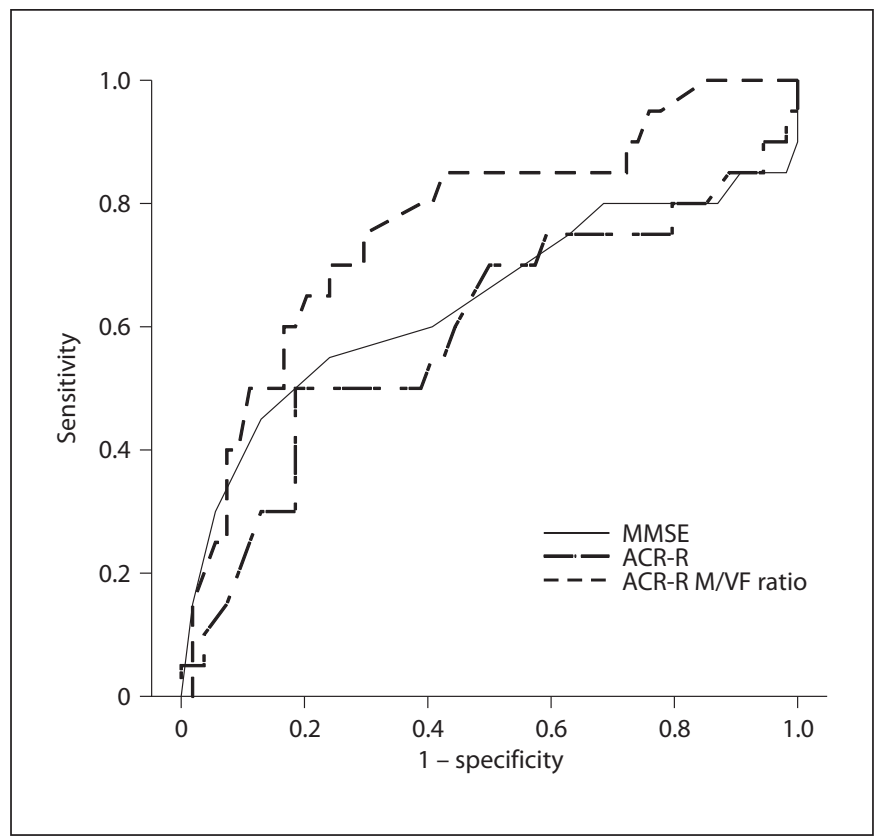

Fig. 4. ACE-R M/VF ratio, ACE-R and MMSE ROC for the distinction between patients with mild dementia in FTLD and patients with mild dementia in $\mathrm{AD}$.

periority of the ACE-R to MMSE in discriminating between healthy controls and patients with the frontal variant of FTLD (AUC: 0.96 vs. 0.92). Such analyses were not performed for patients with the language variant of FTLD due to the limited subsample size.

\section{Distinction between Patients with Mild Dementia in \\ FTLD and Patients with Mild Dementia in AD}

The memory to verbal fluency (M/VF) ratio was calculated since the FTLD and AD groups differed significantly with regard to memory and verbal fluency ACE-R subtest scores. Its value was significantly higher in the FTLD group than in the AD group [mean (SD): 4.13 (2.77) vs. 2.18 (2.07), $t=-2.87, p=0.01]$. The logistic regression analysis (likelihood ratio $\chi^{2}=9.91, \mathrm{p}=0.02$ ) with clinical diagnosis (FTLD vs. AD) as dependent variable and ACE-R M/VF ratio (regression coefficient $=0.35, \mathrm{p}=$ 0.01 ), ACE-R total score (regression coefficient $=0.03$, $\mathrm{p}=0.35$ ) and MMSE score (regression coefficient $=-0.06$, $\mathrm{p}=0.61)$ as independent factors revealed that only the ACE-R M/VF ratio is a significant predictor of the clinical diagnosis. According to the ROC, the ACE-R M/VF ratio (AUC $=0.77$, optimal cut-off score $=2.30 / 2.31$, sensitivity $=0.70$, specificity $=0.76$ ) was shown to be more useful in the distinction between FTLD and AD with fair 
accuracy than the ACE-R total score (AUC $=0.60)$ or the MMSE (AUC $=0.64$ ) (fig. 4), the accuracy of which was poor. The AUCs of the MMSE and the ACE-R total score were not significantly different from 0.5 ( $p>0.05)$, a value indicating that the results are not better than chance at predicting the presence of FTLD or AD.

\section{Discussion}

The role of neurocognitive examination is central in the diagnostic workup of cognitive impairment in ageing. The present study was performed to evaluate the accuracy of the German version of the ACE-R in the distinction between MCI, mild dementia in AD, FTLD, and cognition in normal ageing.

The ACE- $\mathrm{R}$ was not found to be more accurate than the MMSE in distinguishing patients with MCI from cognitively healthy controls. The high values of the AUCs for both tests ( 0.83 for the ACE-R and 0.81 for the MMSE) and the significant association between ACE-R and MMSE scores (both $\mathrm{p}<0.0001$ ) with the clinical diagnosis according to the regression analysis, implying a good accuracy, should be interpreted with caution, since the MMSE has not been proven to be effective in identifying mild cognitive deficits $[15,16]$. These findings possibly reflect a common problem in the recruitment of elderly control subjects. Such volunteers typically represent a selected group of very healthy and highly functioning elderly, differing from the general elderly population, and especially from the elderly individuals referred for dementia evaluation [10,31-33]. Studies of distinct cognitively impaired and cognitively normal samples maximize test performance characteristics [34]. Thus, from the clinician's perspective, emphasis should be placed on the absence of ACE-R superiority to the MMSE in distinguishing healthy controls from patients with MCI.

According to our findings, both the German ACE-R and the MMSE have very high accuracy in detecting mild dementia in AD according to the ROC analysis (both AUCs $=0.99$ ). These extremely high levels of accuracy should, however, be treated with caution, owing to the findings of a recent review study which did not point out such a high utility of the MMSE in detecting patients with dementia in AD [35]. The ACE-R scores were significantly associated with the clinical diagnosis according to the logistic regression analysis $(p=0.02)$. Since the ACE- $R$ encompasses features of learning materials over a series of trials and a delayed recall component, it was expected to be superior to the MMSE in identifying mild dementia in AD. Nevertheless, the shorter MMSE with a p value of 0.05 was shown to tend to predict the clinical diagnosis with high accuracy and to be equally effective in the detection of patients with mild dementia in $\mathrm{AD}$ as the longer ACE-R according to the ROC analysis. Our findings are in line with the observation that no brief cognitive test has yet been found to be clinically superior over others in identifying $\mathrm{AD}$ [4]. In the light of the current short consultation times, the equal effectiveness of the two instruments to detect mild dementia in AD may act as a disincentive to clinical usage of the ACE-R.

ACE-R was found superior to MMSE in detecting mild dementia in FTLD according to the regression and ROC analyses. Though the AUCs of the two instruments differed (AUC $=0.97$ vs. 0.92), the difference did not reach statistical significance. The absence of statistical significance can be explained by the relatively limited sample size of the FTLD group. The superiority of the ACE-R can be attributed to its component tests that assess cognitive domains affected by FTLD (i.e. executive function, verbal fluency) [36] but are not covered by the MMSE. The regression analysis revealed a highly significant association between the clinical diagnosis and the scores of the verbal fluency ACE- $\mathrm{R}$ component test $(\mathrm{p}=0.01)$ and the language component test $(p=0.02)$, which are not or only to a limited extent assessed by the MMSE.

The ACE- $R$ assesses a broad range of cognitive abilities and provides a wide profile of cognitive functions/ dysfunctions. It contributes to drawing a differentiated picture of cognitive deficits with the objective of supporting diagnosis and differential diagnosis. In the present study, the comparison of the AD and the FTLD groups on the ACE-R component tests revealed differences in memory and verbal fluency performance. $\mathrm{AD}$ patients performed better in verbal fluency tasks, whereas the FTLD patients' performance in memory tasks was significantly higher. These observations are concordant with the discrepancies of the profiles of cognitive impairment in these two clinical entities that are highlighted in the literature. For similar levels of overall cognitive decline, FTLD patients have a relative preservation of memory and greater deficits in frontal functioning than $\mathrm{AD}$ patients $[36,37]$. Calculating the ACE-R M/VL ratio further supports with fair accuracy $(\mathrm{AUC}=0.77)$ the differentiation between AD and FTLD.

The German ACE-R was found to detect mild dementia in FTLD more effectively and to differentiate, with the ACE-R M/VF ratio, between FTLD and AD patients significantly better than the MMSE, whilst the accuracy of the two instruments in detecting MCI and mild dementia 
in AD did not differ. Since ACE-R takes around 15 min to perform, whereas administering the MMSE lasts only approximately $8 \mathrm{~min}$ [38], the thorny question is posed about the clinical and diagnostic concept in terms of which the ACE-R could be most appropriately deployed. Measuring a broad range of cognitive abilities and comprising tasks and questions of variable difficulty, the ACE-R provides an objective and differentiated global profile of cognitive performance. This could serve in specialist centres/clinics as an invaluable instrument in a comprehensive diagnostic workup which also encompasses clinical, behavioural and functional features supplemented by an informant report and neuroimaging [39].

Our study has several limitations. The participants were recruited at university centres. Applicability and reliability of the ACE-R in community samples require further investigation. The evaluation was confined to patients with $\mathrm{MCI}, \mathrm{AD}$ and FTLD. Hence, we were not in the position to assess the effectiveness of the ACE-R in detecting other forms of dementia, such as dementia with Lewy bodies or vascular dementia. The reproducibility and repeatability of the ACE-R were not evaluated in this study, but as the ACE-R assesses cognitive functions in a well-standardized and objective manner, the rater-related bias is likely to be low. The statistics of positive and negative predictive values were not calculated, because the groups of patients and healthy controls were recruited independently and not from a population, as well as due to the fact that these values cannot be compared between different diagnostic tests such as the ACE-R and the MMSE [40]. Furthermore, the clinical diagnosis, which was based on a comprehensive diagnostic procedure and on international diagnostic criteria, was used as the ultimate gold standard. In spite of the high validity of the diagnostic criteria, the clinical diagnoses are not always confirmed at autopsy [41]. Thus, the possibly erroneous clinical assessments should also be taken into account. The validity of the ACE-R could therefore be lower than our results suggest.

The diagnosis and differential diagnosis of dementia remain a clinically integrated process, with neurocognitive testing providing only supportive evidence. The ACE-R, a brief and easily administered bedside test, assesses a broad range of cognitive abilities, provides a differentiated objective picture of cognitive impairment and detects patients with mild dementia in FTLD with higher accuracy than the common MMSE. It could emerge as a valid screening instrument that supports clinical and differential diagnosis of dementia, in terms of the clinical and diagnostic concept of specialist centres/clinics.

\section{Acknowledgment}

We thank Dr. Eneida Mioshi (Department of Clinical Neuroscience, University of Cambridge, Cambridge, UK, and Prince of Wales Medical Research Institute, Sydney, N.S.W., Australia) for her assistance in the ACE-R translation into German.

\section{References}

1 Minati L, Edginton T, Bruzzone MG, Giaccone G: Current concepts in Alzheimer's disease: a multidisciplinary review. Am J Alzheimers Dis Other Demen 2009;24:95-121.

2 Chapman DP, Williams SM, Strine TW, Anda RF, Moore MJ: Dementia and its implications for public health. Prev Chronic Dis 2006;3:A34.

3 Harvey RJ, Skelton-Robinson M, Rossor $\mathrm{MN}$ : The prevalence and causes of dementia in people under the age of 65 years. J Neurol Neurosurg Psychiatry 2003;74:1206-1209.

4 Feldman HH, Jacova C, Robillard A, Garcia A, Chow T, Borrie M, Schipper HM, Blair M, Kertesz A, Chertkow H: Diagnosis and treatment of dementia. 2. Diagnosis. CMAJ 2008; 178:825-836.

5 Shulman KI: Clock-drawing: is it the ideal cognitive screening test? Int J Geriatr Psychiatry $2000 ; 15: 548-561$.
6 Jones TG, Schinka JA, Vanderploeg RD, Small BJ, Graves AB, Mortimer JA: 3MS normative data for the elderly. Arch Clin Neuropsychol 2002;17:171-177.

7 Ihl R, Grass-Kapanke B, Lahrem P, Brinkmeyer J, Fischer S, Gaab N, Kaupmannsennecke C: Development and validation of a test for early diagnosis of dementia with differentiation from depression (TFDD). Fortschr Neurol Psychiatr 2000;68:413-422.

8 Kalbe E, Kessler J, Calabrese P, Smith R, Passmore AP, Brand M, Bullock R: Demtect: a new, sensitive cognitive screening test to support the diagnosis of mild cognitive impairment and early dementia. Int J Geriatr Psychiatry 2004;19:136-143.

9 Perry RJ, Hodges JR: Relationship between functional and neuropsychological performance in early Alzheimer disease. Alzheimer Dis Assoc Disord 2000;14:1-10.
10 Alexopoulos P, Nadler K, Cramer B, Herpertz SC, Kurz A: Validation of a short test (3MS-R) for detecting Alzheimer's disease. Fortschr Neurol Psychiatr 2007;75:728-736.

11 Folstein MF, Folstein SE, McHugh PR: Minimental state. A practical method for grading the cognitive state of patients for the clinician. J Psychiatr Res 1975;12:189-198.

12 Mohs RC, Rosen WG, Davis KL: The Alzheimer's disease assessment scale: an instrument for assessing treatment efficacy. Psychopharmacol Bull 1983;19:448-450.

13 Rosen WG, Mohs RC, Davis KL: A new rating scale for Alzheimer's disease. Am J Psychiatry 1984;141:1356-1364.

14 Burkart M, Heun R, Maier W, Benkert O: Dementia screening in routine clinical practice. A comparative analysis of MMSE, SIDAM and ADAS. Nervenarzt 1998;69: 983-990. 
15 Boustani M, Peterson B, Hanson L, Harris R, Lohr KN: Screening for dementia in primary care: a summary of the evidence for the US. Preventive Services Task Force. Ann Intern Med 2003;138:927-937.

16 Kurz A, Diehl J, Riemenschneider M, Perneczky R, Lautenschlager N: Mild cognitive disorder. Questions of definition, diagnosis, prognosis and therapy. Nervenarzt 2004;75: 6-15.

17 Mathuranath PS, Nestor PJ, Berrios GE, Rakowicz W, Hodges JR: A brief cognitive test battery to differentiate Alzheimer's disease and frontotemporal dementia. Neurology 2000;55:1613-1620.

18 Mioshi E, Dawson K, Mitchell J, Arnold R, Hodges JR: The Addenbrooke's cognitive examination revised (ACE-R): a brief cognitive test battery for dementia screening. Int J Geriatr Psychiatry 2006;21:1078-1085.

19 Alexopoulos P, Greim B, Kurz A: Brief assessment of cognitive mental status in german: the Addenbrooke's Cognitive Examination (ACE) and the Addenbrooke's Cognitive Examination Revised (ACE-R). Eur J Geriatr 2007;9175-180.

20 Thalmann B, Mönsch AU: The Consortium to Establish a Registry for Alzheimer's Disease. Neuropsychologische Testbatterie. Basel, Memory Clinic Basel, 1997.

21 Reitan RM, Wolfson D: Validity of the Trail Making Test as an indicator of organic brain damage. Percept Mot Skills 1985;8:271-276.

22 Hindmarch I, Lehfeld H, de Jongh P, Erzigkeit H: The Bayer Activities of Daily Living Scale (B-ADL). Dement Geriatr Cogn Disord 1998;9(suppl 2):20-26.

23 Cummings JL, Mega M, Gray K, RosenbergThompson S, Carusi DA, Gornbein J: The neuropsychiatric inventory: comprehensive assessment of psychopathology in dementia. Neurology 1994;44:2308-2314.
24 Dilling $\mathrm{H}$, Mombour W, Schmidt $\mathrm{MH}$ Schulte-Markwort E, Weltgesundheitsorganisation (eds): Internationale Klassifikation psychischer Störungen. ICD-10 Kapitel V (F). Klinisch-diagnostische Leitlinien. Bern, Huber, 1994

25 McKhann G, Drachman D, Folstein M, Katzman R, Price D, Stadlan EM: Clinical diagnosis of Alzheimer's disease: report of the NINCDS-ADRDA work group under the auspices of department of health and human services task force on Alzheimer's disease. Neurology 1984;34:939-344.

26 Neary D, Snowden IS, Gustafson L, Passant U, Stuss D, Black S, Freedman M, Kertesz A, Robert PH, Albert M, Boone K, Miller BL, Cummings J, Benson DF: Frontotemporal lobar degeneration: a consensus on clinical diagnostic criteria. Neurology 1998;51: 1546-1554.

27 Kraemer HC, Taylor JL, Tinklenberg JR, Yesavage JA: The stages of Alzheimer's disease: a reappraisal. Dement Geriatr Cogn Disord 1998;9:299-308.

28 Winblad B, Palmer K, Kivipelto M, Jelic V, Fratiglioni L, Wahlund LO, Nordberg A, Backman L, Albert M, Almkvist O, Arai H, Basun $\mathrm{H}$, Blennow K, de Leon M, DeCarli C, Erkinjuntti T, Giacobini E, Graff C, Hardy J, Jack C, Jorm A, Ritchie K, van Duijn C, Visser P, Petersen RC: Mild cognitive impairment - Beyond controversies, towards a consensus: report of the international working group on mild cognitive impairment. J Intern Med 2004;256:240-246.

29 Perneczky R, Pohl C, Sorg C, Hartmann J Komossa K, Alexopoulos P, Wagenpfeil S, Kurz A: Complex activities of daily living in mild cognitive impairment: conceptual and diagnostic issues. Age Ageing 2006;35:240245.

30 Vergara IA, Norambuena T, Ferrada E, Slater AW, Melo F: StAR: a simple tool for the statistical comparison of ROC curves. BMC Bioinformatics 2008;9:265.
31 Stokholm J, Vogel A, Johannsen P, Waldemar G: Validation of the Danish Addenbrooke's cognitive examination as a screening test in a memory clinic. Dement Geriatr Cogn Disord 2009;27:361-365.

32 Alexopoulos P, Greim B, Nadler K, Martens U, Krecklow B, Domes G, Herpertz S, Kurz A: Validation of the Addenbrooke's cognitive examination for detecting early Alzheimer's disease and mild vascular dementia in a German population. Dement Geriatr Cogn Disord 2006;22:385-391.

33 Alexopoulos P, Perneczky R, Cramer B, Grimmer T, Kurz A: Validation of a short telephone test (T3MS) for the diagnosis of cognitive impairment. Fortschr Neurol Psychiatr 2006;74:329-336.

34 Brodaty H, Low LF, Gibson L, Burns K: What is the best dementia screening instrument for general practitioners to use? Am J Geriatr Psychiatry 2006;14:391-400.

35 Holsinger T, Deveau J, Boustani M, Williams JW Jr: Does this patient have dementia? JAMA 2007;297:2391-2404.

36 Diehl J, Kurz A: Frontotemporal dementia: patient characteristics, cognition, and behaviour. Int J Geriatr Psychiatry 2002;17: 914-918.

37 Bozoki AC, Farooq MU: Frontotemporal lobar degeneration insights from neuropsychology and neuroimaging. Int Rev Neurobiol 2009;84:185-213.

38 Tangalos EG, Smith GE, Ivnik RJ, Petersen RC, Kokmen E, Kurland LT, Offord KP, Parisi JE: The Mini-Mental State Examination in general medical practice: clinical utility and acceptance. Mayo Clin Proc 1996;71: 829-837.

39 Woodford HJ, George J: Addenbrooke's cognitive examination-revised in day-to-day clinical practice. Age Ageing 2008;37:350-351.

40 Peat J, Barton B: Medical Statistics, ed 2. Malden, Blackwell, 2006.

41 Förstl H: Primär kortikale Demenzen. Stuttgart, Thieme, 2000. 